\title{
A Simple and Effective Method of Color Image Quantization
}

\author{
Sergio Márquez -de-Silva, Edgardo Felipe-Riverón, \\ and Luis Pastor Sánchez Fernández \\ Center for Computing Research, National Polytechnic Institute, Mexico \\ Juan de Dios Batiz w/n, Col Nueva Industrial Vallejo, P.O. 07738, Mexico \\ sama06@sagitario.cic.ipn.mx, edgardo@cic.ipn.mx
}

\begin{abstract}
In this paper a new algorithm for color quantization of color digital image is presented. The method is based on finding homogeneous regions of colors with a level of tolerance depending on the CIE2000 metric. A consequence obtained from the results obtained with other quantization methods is that the number of final colors depends very importantly on the characteristics of the image. In this paper is demonstrated that the proposed method of color quantization have better behavior than the Median-Cut and the Principal Component's algorithms.
\end{abstract}

Keywords: Color quantization, Color image segmentation, L*a*b*, CIE2000.

\section{Introduction}

Color quantization is a process that reduces the number of colors in images without loss of quality and of important global information. Pixels in images could have associated 24-bits containing at most $2^{24}=16,777,216$ different colors. These colors are represented as three dimensional vectors, each vector element with 8-bit dynamic range, allowing $2^{8}=256$ different values. These vectors are often called RGB triplets. A smaller set of representative colors of the image is called color palette. Figure 1 shows the general principle used for the color quantization of color images. From left to right, the original image with thousands of colors; the color palette built with a color quantization method, and finally the rebuilt image created with that palette. Normally, in current color quantization methods, the amount of colors in the final image has to be selected by the user. In the proposed method, the amount of final colors in images is computed automatically with the main goal to keep as possible the qualitative description of the image.
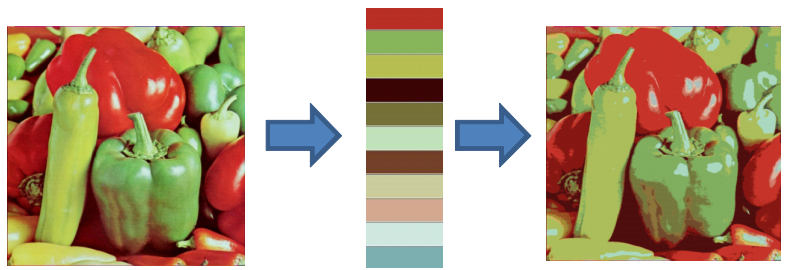

Fig. 1. Color quantization process 


\section{Hypothesis}

The proposed algorithm is compared with two known color quantization methods for the construction of computer graphics color palettes [1]. We conclude that the proposed approach with respect to the Median-Cut and Principal Component's methods permits us to obtain a more acceptable visual representation and more accurate results, allowing the representation of fine details in the quantized image.

\section{State-of-the-Art}

Generally, color quantization involves two steps: choosing or building a proper palette and the reconstruction of the output image using this palette. One straightforward way to obtain quantized images with low computational load consists of using a preselected set of representative colors [2] [3]. Such methods are referred as image-independent quantization methods. Quantized images with higher quality are generally obtained by building the set of representative colors according to the spatial color distribution in the input image. These methods are called adaptive quantization methods or image-dependent quantization methods. Our proposed method falls in the last category. Image-independent color quantization techniques [3] may be viewed as a generalization of the uniform quantization, where the chosen quantization regions are not of the same size but are still independent in the image to be quantized. Such techniques are often used to divide a color space according to a criterion that varies throughout the color space. Due to the complexity of color quantization, preprocessing steps are commonly employed for reducing the data processed by the quantization algorithms. The major techniques used to reduce the data are pre-quantization, which reduces the range of each coordinate in the color space, and the histogram calculation, which allows us to manage the image color set more efficiently. Several methods within this class have been developed. These have been based on clustering methods [4]. In our case, we do not use any pre-quantization algorithm to reduce the data.

\section{Color Representation}

Color models, like all mathematical representations of physical phenomena, can be expressed in many different ways, each with its advantages and drawbacks. Some representation are formulated for helping humans to select colors - the Munsell System [5] for example - and others are formulated to ease data processing in machines, with those used in the RGB space falling in this category. The goal is to minimize formulation complexity and the number of variables, or dimensions. Color perception is a brain process that starts in the eye's cone receptors. These receptors are found in three varieties that exhibit somewhat reddish, greenish, and bluish sensitivities. The "probable" sensitivities can be seen in Hunt [6]. As good as it may be, the CIE 1931 (XYZ model) is not without faults. Soon after it was issued, it was found that it does not represent color gradations in a uniform matter. David L. MacAdam in [7] showed in the early forties that the minimum distance between two 
discernible colors is smaller in the lower left portion, and progressively bigger toward the top. The $\mathrm{L}^{*} \mathrm{a} \mathrm{b}^{*}$ used in our method is derived from the XYZ values. In this color transformation we use the D50 reference white that corresponds to the Daylight, used for color rendering. As it was expected, the CIELAB color space is only approximately perceptually uniform. That is why color difference formulae have been proposed since the acceptance of the CIE standards; most of the difference formulae attempt to use alternate (non-Euclidean) distance measures in the CIELAB space. The CIE2000 color difference formula, denoted as $\Delta E_{00}$, is designed to be symmetric by using averages of the standard and sample colors values in the weighting functions (Eq. 1):

$$
\Delta E_{00}=\sqrt{\left(\frac{\Delta L^{\prime}}{K_{L^{W}}}\right)^{2}+\left(\frac{\Delta C^{\prime}}{K_{c} W_{c}}\right)^{2}+\left(\frac{\Delta H^{\prime}}{K_{H^{W}} W^{2}}\right)^{2}+R_{T}\left(\frac{\Delta C^{\prime}}{K_{c} W_{c}}\right)\left(\frac{\Delta H^{\prime}}{K_{H} W_{H}}\right)}
$$

Where: $\Delta L^{*}, \Delta C^{*}$ and $\Delta H^{*}$ are the differences of luminosity, chrome and hue calculated in the CIELAB model; $W_{L}, W_{C}$ and $W_{H}$ are weighting functions that improve the perceptual uniformity of CIELAB which are calculated as follows (Eq. 2 to 5):

$$
\begin{gathered}
W_{L}=1+\frac{0.015\left(\bar{L}^{\prime}-50\right)^{2}}{\sqrt{20+\left(L^{\prime}-50\right)^{2}}} \\
W_{C}=1+0.045 \bar{C}^{\prime} \\
W_{H}=1+0.015 C^{\prime} T
\end{gathered}
$$

Where

$$
T=1-0.17 \cos \left(H^{\prime}-30^{\circ}\right)+0.24 \cos \left(2 H^{\prime}\right)+0.32 \cos \left(3 H^{\prime}+6^{\circ}\right)-0.2 \cos \left(4 H^{\prime}-63^{\circ}\right)
$$

$K_{L}, K_{C}$ and $K_{H}$ are three dependent numbers of the parametric effects and with a value of 1 for the reference conditions implemented in this work. The reader is referred to reference [8] for details.

\section{Dithering Technique}

Dithering is a technique used in computer graphics to create the illusion of color depth in images with a limited color palette. There are several algorithms designed to perform dithering. One of the earliest, also one of the most popular, is the FloydSteinberg dithering algorithm [9], developed in 1975. One of the strengths of this algorithm is that it minimizes visual artifacts through an error-diffusion process. The Floyd-Steinberg algorithm typically produces images that are closer to represent the original than any simpler dithering algorithms. It is commonly used by image manipulation software, for example, when an image is converted into GIF format that is restricted to a maximum of 256 colors. The algorithm achieves dithering by 
diffusing the quantization error of a pixel to its neighboring pixels, according to a defined distribution. Each time the quantization error is transferred to the neighboring pixels, while not affecting the pixels that have already been quantized. Hence, if a number of pixels have been rounded downwards, it becomes more likely that the next pixel is rounded upwards, so that on average, the quantization error is close to zero. Furthermore, some of them put "ghosts" into the picture. The "ghosting" problem can be ameliorated choosing the coefficients less than 1 .

\section{Description of the Method}

An algorithm has been developed that, contrary to the classical methods for color quantization, does not require to define a priori the size of the color palette. This leads us to an unsupervised method of color quantization. Our method of color quantizing has four phases.

1. Convert the image from the RGB color space to the $\mathrm{L}^{*} \mathrm{a} * \mathrm{~b} *$ color space.

2. Grow regions with the local color average.

3. Reduce the number of colors between regions.

4. Convert the resulting image from the $\mathrm{L}^{*} \mathrm{a} * \mathrm{~b}$ color space to the RGB color space.

In the first phase we convert the RGB color image to the $\mathrm{L}^{*} \mathrm{a} * \mathrm{~b} *$ color space. This nearly perceptually uniform color space distributes the colors in such appropriate way that the human visual system can differentiate all them in the same form. The second phase is focused to obtain color homogeneous regions in the image using a growing process which, starting from a pixel that does not belongs to a region, progressively agglomerates those pixels around that satisfying an homogeneity criterion called by us tolerance threshold. This threshold is given by the user and works as the maximum distance tolerated when the $\Delta E_{00}$ difference is calculated (Eq. 1). The method proposes in this stage a tolerance threshold equal to 8 as a unique value for all type of image. An investigation of the number of colors produced as a function of the tolerance threshold is shown in Table 1. We can observe the initial amount of colors

Table 1. Amount of colors obtained with several values of the tolerance thresholds TT. The images used are shown in Fig. 4, Section 6.

\begin{tabular}{lcccccc}
\hline TT & Cartoon & Lena & Baboon & Paint & Mexico map & Text \\
\hline $\mathbf{0}$ & 398 & 71068 & 256 & 306234 & 144998 & 159 \\
$\mathbf{2}$ & 271 & 648 & 234 & 651 & 1757 & 11 \\
$\mathbf{4}$ & 121 & 83 & 131 & 250 & 187 & 8 \\
$\mathbf{6}$ & 56 & 20 & 52 & 68 & 48 & 7 \\
$\mathbf{8}$ & 39 & 12 & 32 & 24 & 23 & 5 \\
$\mathbf{1 0}$ & 30 & 7 & 17 & 14 & 13 & 5 \\
$\mathbf{1 2}$ & 23 & 5 & 15 & 8 & 10 & 3 \\
$\mathbf{1 6}$ & 13 & 2 & 6 & 7 & 5 & 3 \\
$\mathbf{2 0}$ & 11 & 2 & 4 & 4 & 4 & 3 \\
$\mathbf{2 5}$ & 6 & 1 & 2 & 3 & 2 & 3 \\
$\mathbf{3 0}$ & 4 & 1 & 1 & 3 & 2 & 2 \\
\hline
\end{tabular}


of each image when the tolerance threshold is equal to 0 , and the number of colors obtained after quantization for different values of the tolerance threshold.

The tolerance threshold selected is a balance between the amounts of colors in the images analyzed. The colors grouped change the color region used to agglomerate successive pixels. It is given by the centroid of the pixels clustered in a region in the CIELAB space. Informally, it is the arithmetic average of the colors of all the region points. It is common to explore the images successively line by line or column by column. However, it is worth to note as an interesting result that in our case the scanning of the image was carried out in zigzag. In Figure 2 it is shown at the left a test image with gradually distributed gray levels.
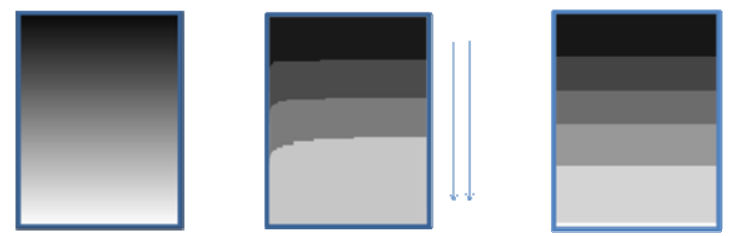

Fig. 2. A test image and their scan versions (a) Original (b) Draining effect (c) With zigzag scan

When the test image is explored column by column or row by row, as in the image of the center (Fig. 2), a draining effect appears in the regions built by the quantization algorithm. This effect is explained schematically in Fig 3. In (A) the original image; (B) Two pixels are grouped generating the first region, which takes the value 3 given by the average of both pixels; (C) The third pixel is grouped to the first region and the fourth pixel is the seed for the second region; (D) and (E) The fifth and sixth pixels are grouped to the first region acquiring this region now the value 4; (F) The seventh pixel have two regions to be grouped, choosing the second one because its value is nearest. $(\mathrm{G})$ All pixels in the image were finally grouped in two regions.

We can observe how the draining effect appears. When it is explored in zigzag traveling to the right, the draining effect disappears. This demonstrates that the algorithm is able to detect details locally in images due to the particular way of regions clustering carried out. We considered in this phase the following premises: (1) the size of the region is calculated by counting the number of pixels belonging to the region. The connectivity considered is the 8-neighbor one; (2) the pixels to be reassigned chose the neighbor region that have a greater influence on it. The influence

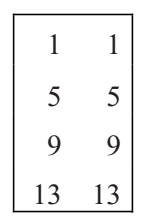

A

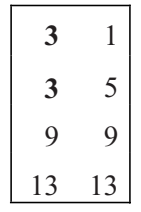

B

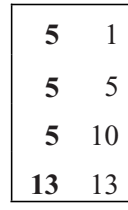

C

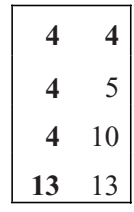

D

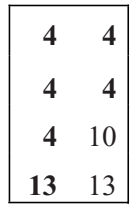

E

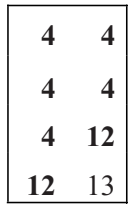

F

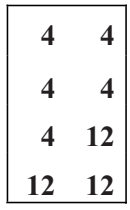

G

Fig. 3. Steps of the region growing phase (by columns) 
is given by the closeness of the pixel values of all neighbor regions. This phase is implemented according to the following pseudo-code listing:

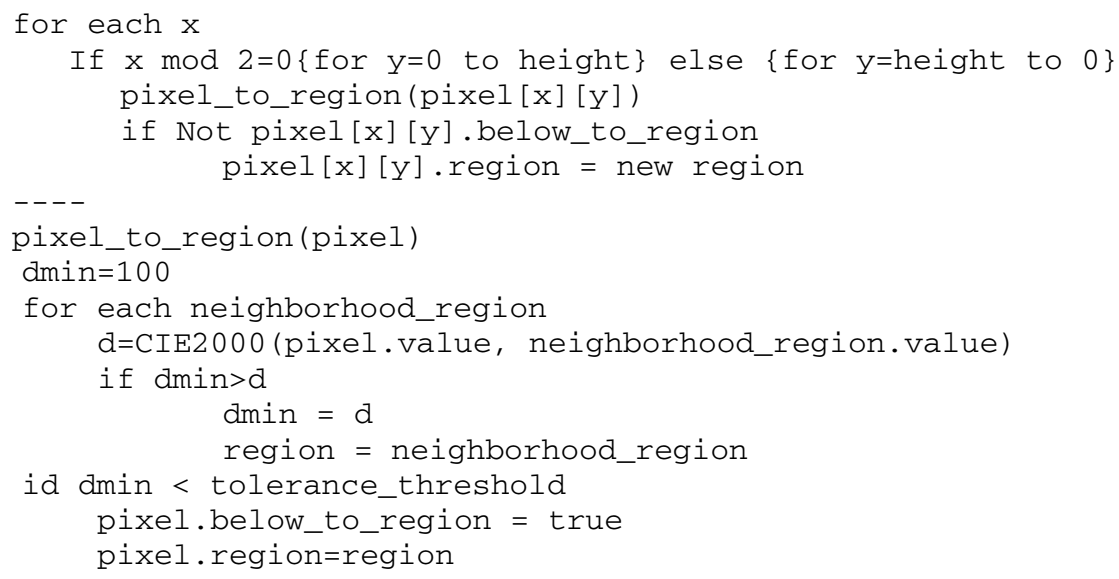

The third phase sorts the regions from bigger to smaller according to the amount of pixels grouped. The smaller regions are marked if they can be grouped to a bigger region using again the tolerance threshold and the CIE2000 metric. The colors of the non-marked regions are used as a palette for rebuilding the image. The pseudo-code of this phase is the following:

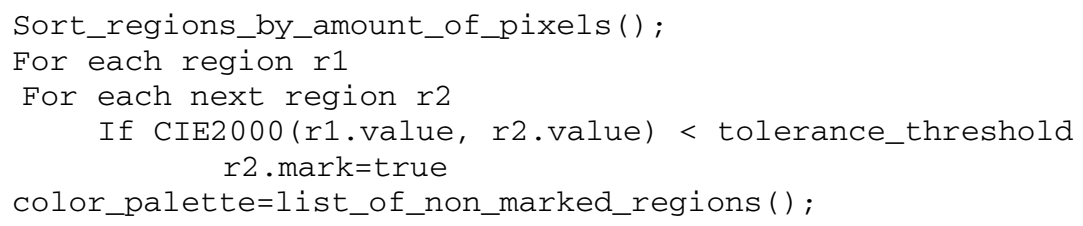

The colors mapping is carried out in the fourth phase using the dithering method explained before (Section 4); finally the output image is built transforming the L*a*b* image to a new RGB image.

\section{Results and Discussion}

To determine the effectiveness of our quantization algorithm, we have compared eight color digital images; two of them are a zoom of a part of these images. To achieve consistent results and to make an appropriate and valid comparison, the following procedure was carried out: (1) To execute our proposed quantization method and observe the amount of colors obtained with it; (2) To adjust the two classical quantization algorithms (Median-Cut and Principal Components) to the amount of colors that were obtained with the proposed method; (3) Use a quadratic error to evaluate the results using the CIE2000 formula like the difference function (Eq. 6).

$$
\text { CIESE }=\frac{1}{M N} \sum_{i=1}^{M} \sum_{j=1}^{N} \operatorname{CIE2000}(c[i, j], q(c[i, j]))^{2}
$$


Figure 4 shows the images used to evaluate the algorithm and Figure 5 show graphically the results detailed numerically in Table 1. The Baboon and Lena images were taken out from the Signal and Image Processing Institute of the University of Southern California (SIPI -USC) database [10] and the Mexico map is a scholar book image chosen by us as a representative image with a large number of small colored graphical symbols and numerous alphanumerical characters. The map of Mexico is a representative image of a problem solved with the proposed methodology to facilitate the recognition of the cartographic symbols. In all cases we used a tolerance threshold value of 8 for the proposed method.
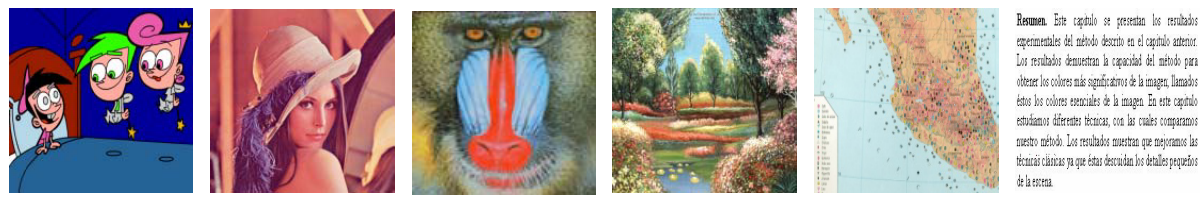

Fig. 4. Images used. From left to right: Cartoon, Lena, Baboon, Paint, Mexico map and Text.
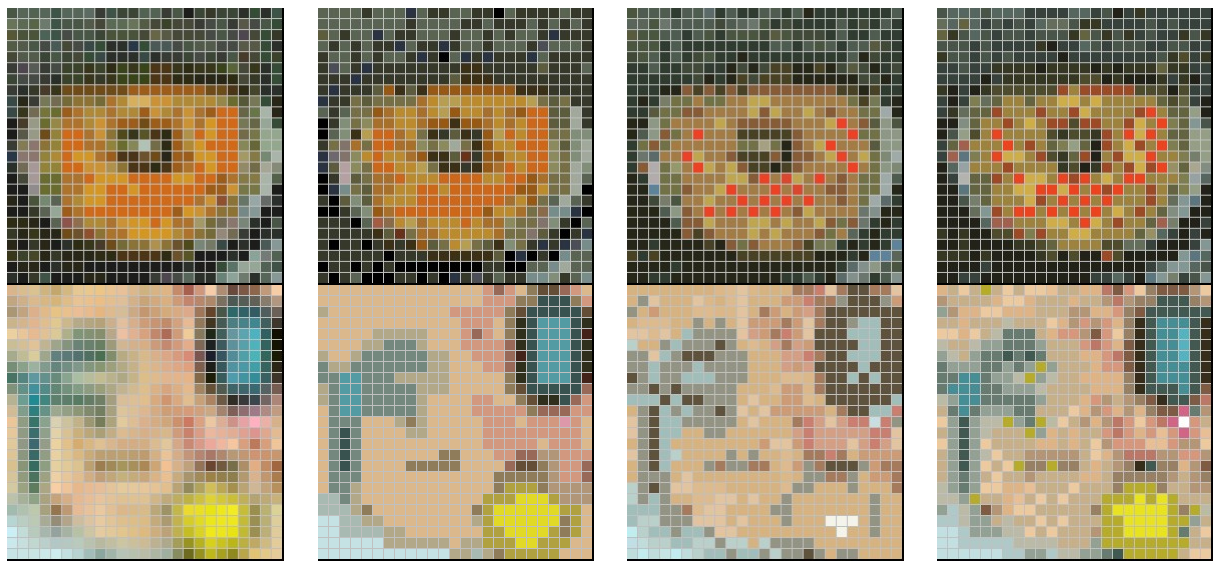

Fig. 5. Zoom of two images with their respective results. From up left to right: Original, Proposed method, Median Cut and Principal Components.

In Fig. 5 appear a zoom of one eye of the Baboon image and a graphical symbol (yellow lemon) of the Mexico map. In the first one it can be appreciated how the proposed quantization method is nobler with the details, because it preserves better the relationship of tones than with any of the other two classical methods. In the second one the algorithm Median-Cut significantly deteriorates the image (disappear the yellow symbol), while the two other methods preserves it, but with better results with the proposed method. Our method is better than the others ones in almost all the cases as shown in Fig 6; only with the text image we observed that the proposed method is worst ( 5 colors with a tolerance threshold of 8 ), but the error is minimal. 


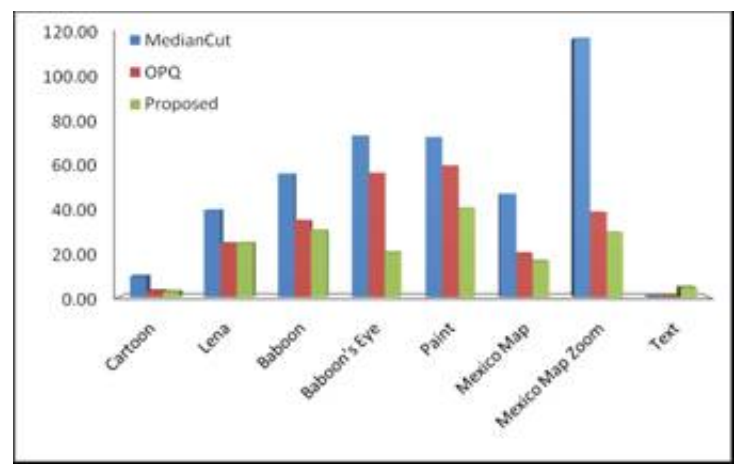

Fig. 6. Results obtained after the application of the quantization process

\section{Conclusion}

In this paper a new color quantization method has been proposed to find homogeneous regions in color images. The only parameter required by the method, a color tolerance threshold, simply is set according to the user desires or priorities. In our examples it was set to eight. The new method does not require the previous definition of the final number of colors to which they will be reduced in the image, like in the other algorithms analyzed. Besides this, the dithering technique of Floyd-Steinberg that increases appreciably the perceptual result has been used by all the techniques evaluated to enhance the quality of images after the color quantization.

Acknowledgments. The authors of this paper wish to thank the Center for Computing Research (CIC), Mexico; Research and Postgraduate Secretary (SIP), Mexico, and National Polytechnic Institute (IPN), Mexico, for their support.

\section{References}

1. Heckbert, P.: Color image quantization for frame buffer display. B. S. thesis, Architecture Machine Group. MIT, Cambridge (1980)

2. Gentile, R., Allebach, J., Walowit, E.: Quantization of color images based on uniform color spaces. J. Imaging Technol. 16(1), 11-21 (1990)

3. Paeth, A.W.: Mapping RGB triples onto four bits. In: Glassner, A.S. (ed.) Graphics Gems, vol. 718, pp. 233-245. Academic Press, Cambridge (1990)

4. Anderberg, M.E.: Cluster Analysis for Applications. Academic Press, N.Y (1973)

5. Birren, F. (ed.): Munsell: A Grammar of Color, A Basic Treatise on the Color System of Albert H Munsell. Van Nostrand Reinhold, New York (1969)

6. Hunt, R.W.G.: The reproduction of Colour, vol. 5. Fountain Press (1995) ISBN 0-86343$381-2$ 
7. MacAdam, D.L.: Visual sensitivities to color differences in daylight. J. Opt. Soc. Am. 32, 247-273 (1942)

8. Luo, M.R., Cui, G., Rigg, B.: The development of the CIE 2000 colour difference formula: CIEDE 2000. Color Res. Appl. 26(5), 340-350 (2001)

9. Floyd, R.W., Steinberg, L.: An adaptive algorithm for spatial gray scale. In: SID (ed.) Int. Symp. Dig. Tech. Papers, vol. 36 (1975)

10. Signal and Image Processing Institute of the University of Southern California (2006), http://sipi.usc.edu/services/datbase/Database.html 